\title{
Predicting the complicated neutropenic fever in the emergency department
}

\author{
J M Moon, B J Chun
}

Department of Emergency Medicine, Chonnam National University Hospital, Gwangju, South Korea

Correspondence to: Dr B J Chun, Department of Emergency Medicine, Chonnam National University Hospital, Hak-Dong 8, Donggu, Gwangju 501-747, South Korea; drmjm@ hanmail.net

Accepted 8 March 2009

\begin{abstract}
Objectives: The purpose of this study was to identify independent factors that can be used to predict whether febrile neutropenic patients who appear healthy at presentation will develop subsequent complications, using variables that are readily available in the emergency department (ED).
\end{abstract}

Method: The medical records of 192 episodes in which the patients presented to the ED with neutropenic fever resulting from chemotherapy, with an alert mental state and haemodynamic stability were retrospectively reviewed. Endpoints examined were fever response to administered antibiotics, death or severe medical complications during hospitalisation.

Results: Thirty-eight episodes of neutropenic fever with complicated outcomes were identified from among a total of 192 episodes. Three parameters emerged as independent factors for the prediction of neutropenic fever with complications in the multivariate regression analysis: platelet count $\left(130-450 \times 10^{3}\right.$ cells $\left./ \mathrm{mm}^{3}\right)<50000$ cells $/ \mathrm{mm}^{3}$, serum C-reactive protein (CRP, $0.1-1 \mathrm{mg} / \mathrm{dl})>10 \mathrm{mg} / \mathrm{dl}$ and pulmonary infiltration on chest $x$ ray.

Conclusions: Platelet count, CRP and pulmonary infiltration on chest $x$ ray at presentation could be used to identify febrile neutropenic patients who will develop complications, and these factors may be useful in making treatment-related decisions in the ED.

Large studies have shown that neutropenic fever after chemotherapy causes death in $4-30 \%$ of patients and serious medical complications in $21 \%$ of patients. ${ }^{12}$ However, it has been suggested that patients with febrile neutropenia are a heterogenous group in terms of medical complications and mortality. Recent advances in the treatment of neutropenic fever have highlighted the value of risk stratification and recommended outpatient treatment for low-risk patients. ${ }^{1-6}$

The degree and duration of neutropenia have been identified as key factors related to the risk and outcome of infection. ${ }^{4}$ However, this finding was of little use to the treating physician because these factors can only be determined retrospectively.

Risk prediction models, including the Talcott model and the Multinational Association of Supportive Care of Cancer risk index scoring system, were developed. ${ }^{13}$ However, the Talcott model requires information, such as tumour response to chemotherapy, which is not readily available in the emergency department (ED). ${ }^{1}$ It is possible that the patient's medical records may not be available in the ED because the patient may be new to the institution. The Multinational Association of Supportive Care of Cancer risk index scoring system includes variables that may be graded differently by different physicians, such as tumour burden of illness.

Previous studies identified clinical or laboratory parameters that could serve as independent predictive factors for risk assessment, including age, acute leukaemia and white blood cell count. ${ }^{7-9}$ However, there is a slight limitation in that these factors were not selected as predictive factors in the other studies for the following reasons. First, the conclusions drawn from studies in children cannot be applied to adults as a result of the differences in comorbidity and the dose of chemotherapy administered. Second, the endpoints used in previous studies, such as bacteraemia, serious bacterial infection or clinical complications, were various.

Studies were recently conducted to analyse the usefulness of serum inflammatory markers, such as procalcitonin, IL-6 and C-reactive protein (CRP), in the assessment of the risk of febrile neutropenia. ${ }^{10-13}$ However, these studies were primarily concerned with determining whether these inflammatory markers can identify bacteraemia rather than subsequent complications, and the value of the markers in predicting patient outcomes is limited. ${ }^{10-13}$

Physicians will regard patients with neutropenic fever combined with shock at presentation as being in a critical condition and treat them intensively. It is thus important to avoid undertreating patients with complicated neutropenic fever, even though they may have appeared healthy upon presentation at the ED.

However, to our knowledge, no study has been conducted to identify the objective independent variables that can be easily assessed in the ED and use them to predict which patients with febrile neutropenia and haemodynamic stability at presentation will develop subsequent potentially serious complications.

Therefore, we sought to identify simple independent factors that can predict patients who develop subsequent complications after chemotherapy-induced neutropenic fever.

\section{METHODS}

\section{Design}

The present study was a retrospective cohort study that was conducted using chart review methodology. This study was approved by our hospital's institutional review board.

\section{Setting and population}

This study was conducted at a single academic tertiary care centre with an annual ED census of 40000 patients with or without cancer per year. online under the BMJ Journals unlocked scheme, see http:// emj.bmj.com/info/unlocked.dtl 
The hospital is a large urban medical centre and is designated as one of five specialised cancer centres in South Korea.

We used the hospital electronic medical record system to obtain the medical record numbers of the patients enrolled in this study. The patient selection criteria were as follows: patients presenting to the ED with C00-C97 codes (malignant neoplasm) classified according to the International Classification of Diseases, version 10 between March 2004 and December 2007, neutrophil count less than 500 cells $/ \mathrm{mm}^{3}$, age older than 18 years and systolic blood pressure greater than $90 \mathrm{~mm} \mathrm{Hg}$ at presentation. Fever was defined as an oral temperature greater than $38.3^{\circ} \mathrm{C}$ on a single occasion or greater than $38.0^{\circ} \mathrm{C}$ for one hour or longer during the first $24 \mathrm{~h}$ after admission. ${ }^{14}$ A total of 254 episodes was identified. However, six episodes presenting to the $\mathrm{ED}$ in an altered mental state were excluded. In addition, we excluded nine episodes who underwent radiotherapy before or during the febrile neutropenic episode, 27 episodes who were transferred to other hospitals or our hospital in the middle of treatment for neutropenic fever, 11 episodes who presented with neutropenic fever as an initial clinical manifestation of unevaluated underlying cancer, seven episodes with incomplete data and two episodes with AIDS.

Finally, 192 febrile neutropenic episodes occurring in 168 patients were analysed. Each febrile neutropenic episode was considered to be a new event.

\section{Protocol}

The first author assessed whether the patient met the defined criteria for combined fever and chemotherapy-related neutropenia. The first assistant, who was unaware of the purpose of the study, classified each patient as with or without risk of complications during hospitalisation. Later, a second author, who was unaware of the risk classification and had previous experience with data abstraction from electronic medical records, collected the variables such as initial laboratory findings, empirically administered antibiotics and duration of hospitalisation. To test the accuracy of the risk assessment by the first assistant, a second assistant, who was not listed as an author, evaluated $15 \%$ of the patient medical records selected by random sampling. There was no disagreement on the risk assignment.

Complicated neutropenic fever was defined when the fever did not resolve within 5 days of starting treatment, in case of death, or in patients with serious medical complications that needed treatment and occurred during hospitalisation.

\section{Measurement}

The following data were collected: (1) variables that were readily available at presentation without information about the medical history of the patient and tested for their predictive value, such as age, sex, tumour type, laboratory variables and the interpretation of chest $x$ ray by a radiologist; (2) other variables related to patients, such as latency to the development of neutropenic fever after recent chemotherapy, previous medical history, prophylactic administration of antibiotics or antifungal agents and presumed site of infection at presentation; (3) variables related to treatment and outcome, including empirically administered antibiotics, microbiological test results, duration of neutropenia and fever, complications, duration of hospitalisation and survival.

The patients were divided according to microbiologically defined infection with or without bacteraemia, clinically defined infection and unexplained fever according to previously published definitions. ${ }^{15}$

\section{Data analysis}

Baseline data on characteristics and outcomes were summarised by frequency tabulation or mean values. Univariate analysis of the association between each covariate and outcome was performed using the $\chi^{2}$ test. Continuous variables tested in the univariate analyses were categorised on the basis of clinical arguments according to physician's judgement or previously published reports. All covariates with $p$ values less than 0.05 were considered sufficient for the inclusion of a variable in multivariate stepwise logistic regression analysis, with a significance level of $p<0.05$. Estimated odds ratios and confidence intervals were calculated for all significant variables. All reported $\mathrm{p}$ values are two-sided, and all statistical analyses were performed using SPSS version 15.0.

\section{RESULTS}

\section{Patient characteristics}

A total of 154 episodes without complications and 38 episodes with serious complications was analysed. Table 1 shows the characteristics and demographic data of the 192 episodes.

The mean age in the study group was 53 years. The mean blood pressure was $126.2 \mathrm{~mm} \mathrm{Hg}$ (SD 16.8) and the temperature at presentation was $37.9^{\circ} \mathrm{C}$ (SD 1.0). Fifty-nine episodes had haematological malignancies, of which lymphoma (47 episodes) was the most common underlying haematological malignancy, followed by 11 cases of leukaemia and one case of multiple myeloma.

All patients were admitted and treated with empiric broadspectrum antibiotics as inpatients. A single antibiotic (meropenema, imipenem, cefepime or ceftazidime) was administered to 23 episodes. A total of 169 episodes (88.0\%) was treated with

Table 1 Clinical characteristics of the 192 episodes of febrile neutropenia

\begin{tabular}{lc}
\hline Clinical characteristic & Episode \\
\hline Mean age (years) & $53.5($ SD 12.4$)$ \\
Gender (male/female) & $70 / 122$ \\
Underlying cancer, N (\%) & \\
Breast cancer & $65(33.9 \%)$ \\
Haematological cancer & $59(30.7 \%)$ \\
Pulmonary cancer & $24(12.5 \%)$ \\
Gastrointestinal cancer & $23(12.0 \%)$ \\
Skeletal cancer & $6(3.1 \%)$ \\
Hepatobiliary cancer & $3(1.6 \%)$ \\
Other solid tumour & $12(6.3 \%)$ \\
Comorbidity, N (\%) & \\
Recent surgery (<6 months) & $46(24.0 \%)$ \\
Previous neutropenic fever & $47(24.5 \%)$ \\
Diabetes mellitus & $19(9.9 \%)$ \\
Heart failure & $5(2.6 \%)$ \\
Prophylactic medication, N (\%) & \\
Antibiotic medication & $24(12.5)$ \\
Antifungal medication & $6(3.1 \%)$ \\
Central venous catheter in place, N (\%) & $11(5.7 \%)$ \\
First installed chemotherapy, N (\%) & $61(31.8 \%)$ \\
Latency after chemotherapy $<7$ days, N (\%) & $41(24 \%)$ \\
Duration of fever at presentation $<24 \mathrm{~h}, \mathrm{~N}(\%)$ & $135(70.3 \%)$ \\
Presumed site of infection at presentation, N (\%) & \\
Gastrointestinal tract & $65(33.9 \%)$ \\
Unexplained fever & $62(32.3 \%)$ \\
Pulmonary tract & $44(22.9 \%)$ \\
Other & $21(10.9 \%)$ \\
\hline
\end{tabular}


Table 2 Serious medical complications developed in patients with complicated neutropenic fever

\begin{tabular}{lc}
\hline Complications & N (\%) \\
\hline Hypotension* & $13(34.2 \%)$ \\
Respiratory failure $\dagger$ & $9(23.7 \%)$ \\
Disseminated intravascular coagulation & $7(18.4 \%)$ \\
Renal failuref & $2(5.3 \%)$ \\
Severe bleeding to require tranfusion & $2(5.3 \%)$ \\
Altered mental state & $1(2.6 \%)$ \\
Arrhythmia requiring treatment & $1(2.6 \%)$ \\
Others & $3(7.9 \%)$ \\
\hline
\end{tabular}

*Hypotension was defined as systolic blood pressure less than $90 \mathrm{~mm} \mathrm{Hg}$ or the need for pressor to maintain blood pressure. $\uparrow$ Respiratory failure was defined as arterial oxygen pressure less than $60 \mathrm{~mm} \mathrm{Hg}$ while breathing room air or the need for mechanical ventilation. $\$$ Renal failure required treatment with fluids, dialysis or any other intervention.

the above-mentioned antibiotics in combination with an aminoglycoside. Eighty-three episodes with neutrophil counts less than 100 cells $/ \mathrm{mm}^{3}$ received haematopoietic growth factors during hospitalisation.

\section{Outcome}

Nine $(5.8 \%)$ of the episodes in the group of patients without complications and eight (21.1\%) of the episodes in the group of patients with complications had a microbiological pathogen $(p=0.001)$. In addition, of the 60 episodes who had an unexplained fever, 57 were in the group of patients without complications $(p=0.001)$. However, there was no difference in the incidence of clinically defined infection between the groups with and without complications.

Neutropenia (2.6 days (SD 2.0) vs 3.8 days (SD 1.9), $\mathrm{p}=0.001$ ) lasted significantly longer in the group of patients with complications than in the group of patients without complications. Patients without complications were discharged 6.6 days (SD 4.1) after hospitalisation, whereas patients with complications were hospitalised for 18.2 days (SD 16.8; $\mathrm{p}<0.001)$.

The most frequent complications were hypotension $(34.2 \%)$, followed by respiratory failure (23.7\%; table 2 ).
These complications occurred within one to 9 days after admission. Other complications deemed clinically significant by the investigator included two cases of electrolyte disturbance and one case of hepatic failure. The fever did not resolve within 5 days after starting treatment in 14 episodes, and seven of them did not show any kind of serious medical complication. Seven $(3.6 \%)$ of the patients died, and each of these patients had at least two serious medical complications.

\section{Risk stratification}

Univariate analysis of the clinical parameters readily available in the ED was performed (table 3).

In the multivariate analysis, platelets less than 50000 cells/ $\mathrm{mm}^{3}$, CRP greater than $10 \mathrm{mg} / \mathrm{dl}$ and pulmonary infiltration on chest $x$ ray were determined to be independent factors for the prediction of neutropenic fever with complications that requires maximal attention from physicians (table 4).

\section{DISCUSSION}

The number of patients who appear healthy at presentation but will develop serious complications and in whom independent variables can predict such an outcome in this group is an important question for emergency medicine physicians. Therefore, we only enrolled patients who were alert and had systolic blood pressure greater than $90 \mathrm{~mm} \mathrm{Hg}$ at presentation. In addition, we observed the elevation of body temperature during the first $24 \mathrm{~h}$ after presentation, in order to include patients who had taken an antipyretic agent before presentation and did not have a body temperature greater than $38.3^{\circ} \mathrm{C}$ at presentation.

In the present study, $19.8 \%$ of episodes experienced at least one serious medical complication or episode of prolonged fever. Early predictive markers of neutropenic febrile patients who are likely to develop serious complications are thus needed to allow the rapid evaluation and treatment of febrile neutropenia, as in $19.8 \%$ of the episodes in our study.

In our study, we excluded parameters that were affected by the subjective judgement of the physician and those that were obtained retrospectively or through detailed analysis of the patient's medical history, even though these factors were

Table 3 Univariate analysis of outcomes for patients with neutropenic fever

\begin{tabular}{|c|c|c|c|c|}
\hline & $\begin{array}{l}\text { Total } \\
\text { N (192) }\end{array}$ & $\begin{array}{l}\text { Without } \\
\text { complications } \\
\text { N (154) }\end{array}$ & $\begin{array}{l}\text { With } \\
\text { complications } \\
\text { N (38) }\end{array}$ & p Value \\
\hline Age $>65$ years & $37(19.3 \%)$ & $28(18.2 \%)$ & $9(23.7 \%)$ & 0.441 \\
\hline Male & $70(36.5 \%)$ & $49(31.8 \%)$ & $21(55.3 \%)$ & 0.007 \\
\hline Haematological cancer & $59(30.7 \%)$ & $41(26.6 \%)$ & $18(47.4 \%)$ & 0.013 \\
\hline \multicolumn{5}{|l|}{ Laboratory findings } \\
\hline WBC $<500$ cells $/ \mathrm{mm}^{3}$ & $83(43.2 \%)$ & $60(39.0 \%)$ & $23(60.5 \%)$ & 0.016 \\
\hline Platelets $<50000$ cells $/ \mathrm{mm}^{3}$ & $46(24.0 \%)$ & $26(16.9 \%)$ & $20(52.6 \%)$ & $<0.001$ \\
\hline Monocytes $<100$ cells $/ \mathrm{mm}^{3}$ & $92(47.9 \%)$ & $66(42.9 \%)$ & $26(68.4 \%)$ & 0.005 \\
\hline Neutrophils $<100$ cells $/ \mathrm{mm}^{3}$ & $115(59.9 \%)$ & $91(59.1 \%)$ & $24(63.2 \%)$ & 0.647 \\
\hline Lymphocytes $<100$ cells $/ \mathrm{mm}^{3}$ & $37(19.3 \%)$ & $25(16.2 \%)$ & $12(31.6 \%)$ & 0.032 \\
\hline Total protein $(6-8.3 \mathrm{~g} / \mathrm{dl})<6.0 \mathrm{~g} / \mathrm{dl}$ & $51(26.6 \%)$ & $36(23.4 \%)$ & $15(39.5 \%)$ & 0.044 \\
\hline Albumin $(3.5-5 \mathrm{~g} / \mathrm{dll})<3.0 \mathrm{~g} / \mathrm{dl}$ & $25(13.0 \%)$ & $17(11.0 \%)$ & $8(21.1 \%)$ & 0.100 \\
\hline BUN $(8-20 \mathrm{mg} / \mathrm{dl})>20 \mathrm{mg} / \mathrm{dl}$ & $32(16.7 \%)$ & $22(14.3 \%)$ & $10(26.3 \%)$ & 0.075 \\
\hline Creatinine $(0.5-1.2 \mathrm{mg} / \mathrm{dl})>1.2 \mathrm{mg} / \mathrm{dl}$ & $19(9.9 \%)$ & $12(7.8 \%)$ & $7(18.4 \%)$ & 0.049 \\
\hline $\operatorname{CRP}(0.1-1.0 \mathrm{mg} / \mathrm{dl})>10 \mathrm{mg} / \mathrm{dl}$ & $78(40.6 \%)$ & $52(33.8 \%)$ & $26(68.4 \%)$ & $<0.001$ \\
\hline Positive test for urine nitrates & $18(9.4 \%) .8 \%)$ & $16(10.4 \%)$ & $2(5.3 \%)$ & 0.332 \\
\hline Pulmonary infiltration & $18(9.4 \%)$ & $3(1.9 \%)$ & $15(39.5 \%)$ & $<0.001$ \\
\hline
\end{tabular}

The normal ranges of complete blood counts are as follows: white blood cells (WBC) $\left(4.8-10.8 \times 10^{3} \mathrm{cells} / \mathrm{mm}^{3}\right)$, platelets $\left(130-450 \times 10^{3}\right.$ cells $\left./ \mathrm{mm}^{3}\right), 2-9 \%$ monocytes, $50-75 \%$ neutrophils and $20-40 \%$ lymphocytes of WBC. BUN, blood urea nitrogen. 
Table 4 Independent predictors identified by multivariate analysis

\begin{tabular}{lllll}
\hline Variable & $\boldsymbol{\beta}$ & $\mathbf{O R}$ & $\mathbf{p}$ Value & \multicolumn{1}{c}{$\mathbf{9 5 \%} \mathbf{C l}$} \\
\hline $\mathrm{CRP}>10 \mathrm{mg} / \mathrm{dl}$ & 1.000 & 2.719 & 0.028 & 1.111 to 6.651 \\
Platelets $<50000 \mathrm{cells} / \mathrm{mm}^{3}$ ) & 1.606 & 4.982 & 0.001 & 2.000 to 12.408 \\
Pulmonary infiltration & 3.407 & 30.167 & $<0.001$ & 7.281 to 124.998 \\
\hline
\end{tabular}

CRP, C-reactive protein; OR, odds ratio.

considered to be predictive factors in previous reports. Finally, platelet count, CRP and pulmonary infiltration were shown to have a significant association with outcome. The clear advantage of these significant variables is that there is no observer variability and they can be very readily assessed on presentation.

The initial complete blood cell count had been investigated in several studies of risk assessment. Studies in the field of paediatrics by Rackoff et $a l^{7}$ Baorto et $a l^{16}$ and Klaassen et a ${ }^{17}$ proposed that the monocyte count with a specific cutoff value was a significant factor in the development of bacteraemia or severe bacterial infection. In a recent prospective study, ${ }^{12}$ none of the haematological parameters were found to be independent variables for the prediction of complications in adults with chemotherapy-induced febrile neutropenia. In our study, only platelets less than 50000 cells $/ \mathrm{mm}^{3}$ was predictive of a complicated neutropenic fever. It may be possible that the platelet count acts as a surrogate marker of the host response to inflammation and exposure to activated coagulation factors to accelerate disseminated intravascular coagulopathy before the initiation of therapy.

The level of serum CRP was also recognised as an independent factor in the multivariate model. CRP was sensitive for the prediction of neutropenic fever with shock or complex infection in haematological malignancies. ${ }^{18}$ It is possible that the production of CRP is stimulated by cytokines in response to infection or inflammation. However, CRP failed to predict deterioration in adults with neutropenic fever subsequent to chemotherapy. ${ }^{11} 12$ These controversial consequences might result from its late response to inflammation and a wide range of serum levels related to the amount of tissue injury.

It is noteworthy that abnormality on chest $x$ ray was significantly associated with outcome, as in several previous studies. ${ }^{3}$ In particular, it was noted in $15-25 \%$ of all episodes of febrile neutropenia after chemotherapy for haematological malignancies, and they could be responsible for the mortality rates of up to $23-50 \%{ }^{19}{ }^{20}$ In our study, pulmonary infiltration had a higher odds ratio than other significant variables.

We suggest that the initial platelet count and serum CRP as well as pulmonary infiltration could be used to identify patients with neutropenic fever who appear well at admission but will develop complications. This may maximise the chance of identifying patients who are most likely to benefit from rapid admission and intensive treatment. We did not insist that the patients without predictive factors should not be admitted. We cautiously suggested outpatient treatment in these cases after observing the patient for 2-24 h to confirm the assessment of risk, assess the patient's medication tolerance and provide patient education, if treatment guidelines in an individual institution are established.

This study has several limitations. First, this study was performed at a single institution, which is one of five specialised cancer centres in our country. Therefore, our findings may not be representative of all other institutions, and they may vary according to the chemotherapy regimen. Second, the results of our study are based on a retrospective analysis of a small population, and should therefore be interpreted with some caution and confirmed through prospective studies. However, there were no missing data regarding the laboratory findings that were not influenced by reviewer bias and inconsistencies in patient medical records. Third, there was no standard guideline for the administration of empirical antimicrobial agents due to the retrospective nature of the study, and the physician's familiarity affected the choice of antimicrobial agent. However, in our study, extended antimicrobial therapy was administered initially and then modified based on microbiological findings and the patient's clinical response. ${ }^{21}$ Fourth, haematopoietic growth factor was administered to 83 patients in our study. However, there was no difference in the administration of haematopoietic growth factor between the two groups, and the benefits of haematopoietic growth factor have not been definitively proved. ${ }^{22}$

Acknowledgements: The authors would like express sincere thanks to GS Lee, MH Sin and Yl Min for supporting them in the study.

Competing interests: None.

Ethics approval: This study was approved by the Chonnam National University Hospital institutional review board.

Contributors: GS Lee and YI Min, who did not know the purpose of the study, classified each episode as with or without complication. MH Sin provided the statistical advice.

Provenance and peer review: Not commissioned; externally peer reviewed.

\section{REFERENCE}

1. Talcott JA, Finberg R, Mayer RJ, et al. The medical course of cancer patients with fever and neturopenia. Clinical identification of a low risk subgroup at presentation. Arch Intern Med 1988;148:2561-8.

2. Schimpff SC. Empiric antibiotic therapy for granulocytopenic cancer patients. Am J Med 1986;80:13-20.

3. Klastersky J, Paesmans M, Rubenstein EB, et al. The Multinational Association for Supportive Care in Cancer risk index: a multinational scoring system for identifying low risk febrile neutropenic cancer patients. J Clin Oncol 2000;18:3038-51.

4. Pizzo PA, Robichaud KJ, Gill FA, et al. Duration of empiric antibiotic therapy in granulocytopenic patients with cancer. Am J Med 1979;67:194-200.

5. Rolston KV, Manzullo EF, Elting LS, et al. Once daily, oral, outpatient quinolone monotherapy for low-risk cancer patients with fever and neutropenia: a pilot study based on validated risk prediction rules. Cancer 2006;106:2489-94.

6. Sebban C, Dussart S, Fuhrmann C, et al. Oral moxifloxacin or intravenous ceftriaxone for the treatment of low-risk neutropenic fever in cancer patients suitable for early hospital discharge. Support Care Cancer 2008;16:1017-23.

7. Rackoff WR, Gonin R, Robinson C, et al. Predicting the risk of bacteremia in children with fever and neutropenia. J Clin Oncol 1996;14:919-24.

8. Viscoli C, Bruzzi P, Castagnola E, et al. Factors associated with bacteremia in febrile, granulocytopenic cancer patients. Eur J Cancer 1994;30A:430-7.

9. Hubel K, Hegener K, Schnell R, et al. Suppressed neutrophil function as a risk factor for severe infection after cytotoxic chemotherapy in patients with acute nonlymphocytic leukemia. Ann Hematol 1999;78:73-7.

10. Jimeno A, Garcia-Velasco A, del Val 0, et al. Assessment of procalcitonin as a diagnostic and prognostic marker in patients with solid tumors and febrile neutropenia. Cancer 2004;100:2462-9.

11. Persson L, Soderquist B, Engervall $P$, et al. Assessment of systemic inflammation markers to differentiate a stable from deteriorating clinical course in patients with febrile neutropenia. Eur J Haematol 2005;74:297-303.

12. Uys A, Rapoport BL, Fickl H, et al. Prediction of outcome in cancer patients with febrile neutropenia: comparison of the Multinational Association of Supportive Care in Cancer risk-index score with procalcitonin, $C$ reactive protein, serum amyloid $A$, and interleukins-1beta, -6, -8 and -10. Eur J Cancer Care 2007;16:475-83.

13. Persson L, Engervall $P$, Magnuson A, et al. Use of inflammatory markers for early detection of bacteraemia in patients with febrile neturopenia. Scand J Infect Dis 2004;36:365-71. 
14. Hughes WT, Armstrong D, Bodey GP, et al. 2002 Guidelines for the use of antimicrobial agents in neutropenic patients with cancer. Clin Infect Dis 2002;34:730-51.

15. Immunocompromised Host Society. The design, analysis, and reporting of clinical trials on the empirical antibiotics management of the neutropenic patient. Report of a consensus panel. J Infect Dis 1990;161:397-401.

16. Baorto EP, Aquino VM, Mullen CA, et al. Clinical parameters associated with low bacteremia risk in 1100 pediatric oncology patients with fever and neutropenia. Cancer 2001;92:909-13.

17. Klaassen RJ, Goodman TR, Pham B, et al. Low risk prediction rule for pediatric oncology patients presenting with fever and neutropenia. J Clin Oncol 2000:18:1012-19.
18. Erten M, Genc S, Besisik SK, et al. The predictive and diagnostic values of procalcitonin and $\mathrm{C}$-reactive protein for clinical outcome in febrile neutropenic patients. J Chin Med Assoc 2004;67:217-21.

19. Offidani M. Corvatta L, Malerba L, et al. Risk assessment of patients with hematologic malignancies who develop fever asscompanied by pulmonary infiltrates: a historical cohort study. Cancer 2004;101:567-77.

20. Heussel CP, Kauczor HU, Ullmann AJ. Pneumonia in neutropenic patients. Eur Radio 2004; 14:256-71

21. Sipsas NV, Bodey GP, Kontoyiannis DP. Perspectives for the management of febrile neutropenic patients with cancer in the 21st century. Cancer 2005;103:1103-13.

22. Garcia-Carboner R, Paz-Aves L. Antibiotics and growth factors in the management of fever and neutropenia in cancer patients. Curr Opin Hematol 2002;9:215-21.

\section{Images in emergency medicine}

\section{Pain in the leg after jogging}

A 59-year-old woman complained of pain in her left knee. She told us that she had been suffering from osteoarthrosis of the left knee and underwent a medial meniscectomy 8 years ago. She also mentioned that she started exercising 8 weeks ago by jogging. The pain started immediately after one jogging session. We performed an $x$ ray and a magnetic resonance imaging scan of her left knee (fig 1), which showed ostoearthrosis of the medial knee compartment and a translucent patchy spot in proximal tibia. The patient was diagnosed as having a stress fracture of her tibia caused by the sudden commencement of jogging. The patient was managed conservatively with an above-the-knee circular polymer resin cast for 6 weeks. The first 2 weeks were non-weight bearing and the remaining 4 weeks were weight bearing. After 6 weeks the patient was symptom free.

\section{S M M de Castro, P Joosse, Ç Ünlü, E P H Steller}

Department of Surgery, Saint Lucas Andreas Hospital, Amsterdam, The Netherlands

Correspondence to: Dr S M M de Castro, Jan Tooropstraat 164, 1061 AE, Amsterdam, The Netherlands; stevedecastro@gmail.com

\section{Competing interests: None.}

Patient consent: Obtained.

Accepted 9 September 2008

Emerg Med J 2009;26:806. doi:10.1136/emj.2008.066605
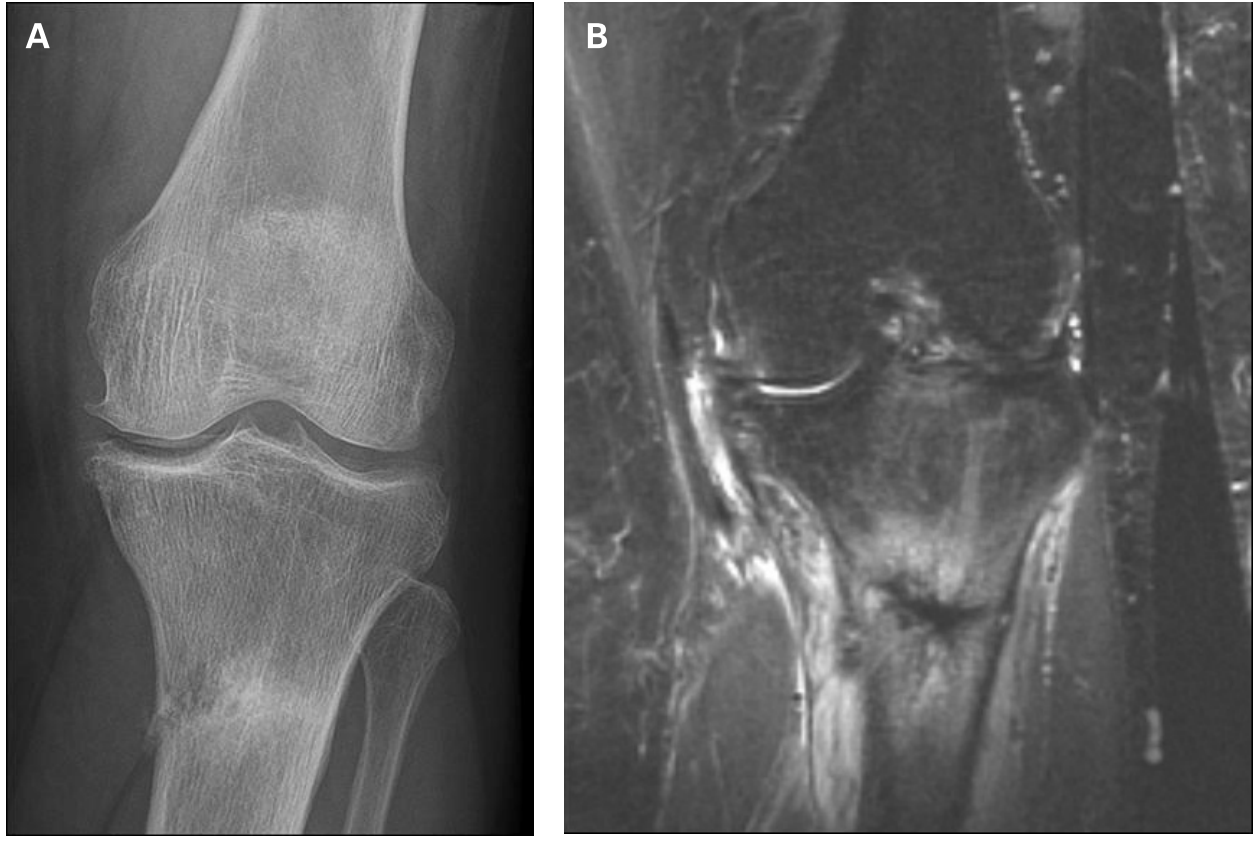

Figure 1 (A) Anterior-posterior $x$ ray of the left knee showing ostoearthrosis of the medial knee compartment. Translucent patchy spot of the proximal tibia. (B) Antero-posterior magnetic resonance imaging scan shows bone marrow oedema and a fracture line. 